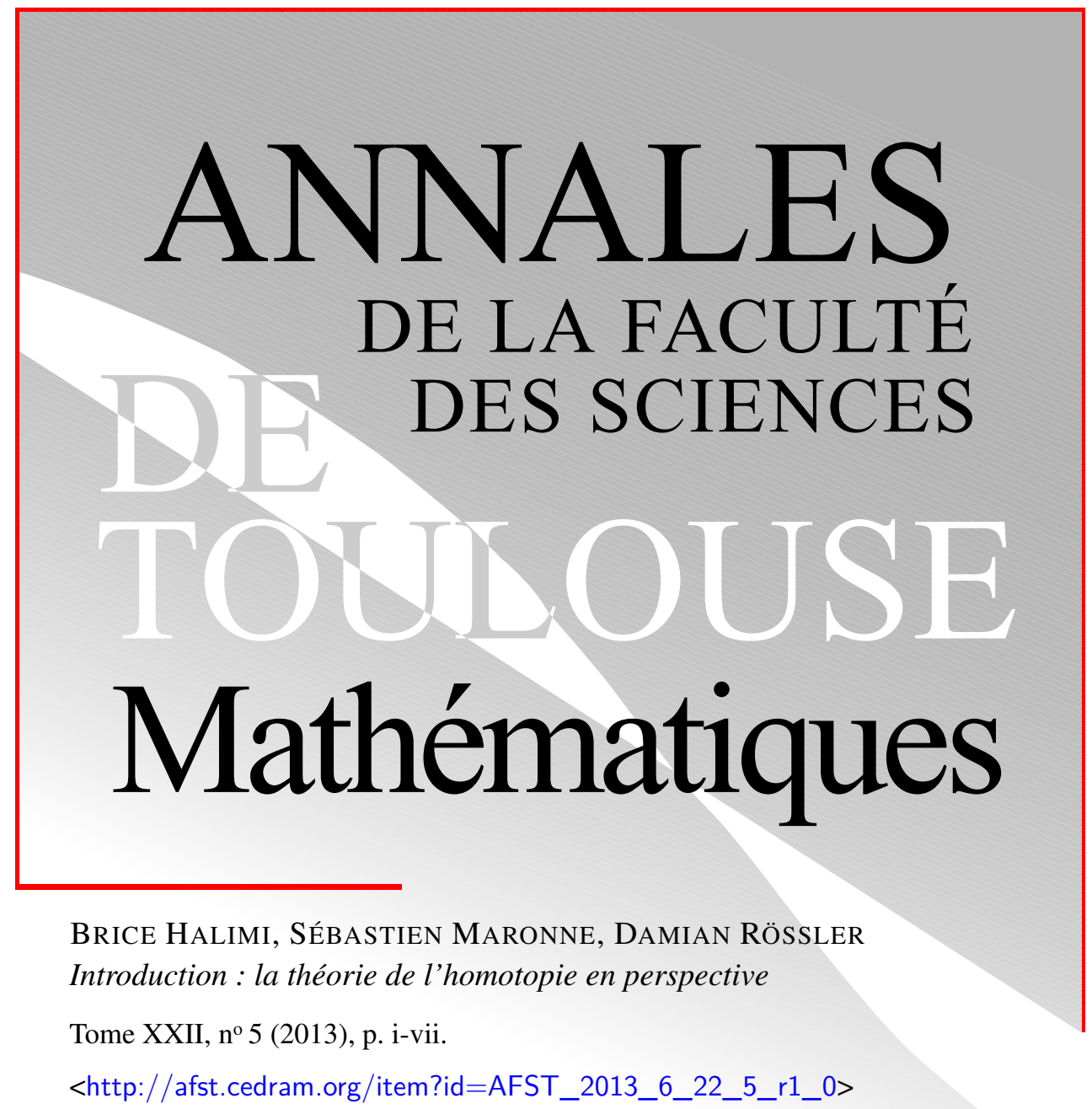

(C) Université Paul Sabatier, Toulouse, 2013, tous droits réservés.

L'accès aux articles de la revue «Annales de la faculté des sciences de Toulouse Mathématiques » (http://afst.cedram.org/), implique l'accord avec les conditions générales d'utilisation (http://afst.cedram.org/ legal/). Toute reproduction en tout ou partie de cet article sous quelque forme que ce soit pour tout usage autre que l'utilisation à fin strictement personnelle du copiste est constitutive d'une infraction pénale. Toute copie ou impression de ce fichier doit contenir la présente mention de copyright.

\title{
cedram
}

Article mis en ligne dans le cadre du

Centre de diffusion des revues académiques de mathématiques

http://www.cedram.org/ 


\section{Introduction : la théorie de l'homotopie en perspective}

Bien que les mathématiques, la logique et la philosophie croisent leurs interrogations depuis leurs origines respectives, des rencontres entre les mathématiques et la philosophie contemporaines sont plus rares. En effet, même si un véritable dialogue entre les mathématiques à l'œuvre et la philosophie a eu l'occasion de se développer par le passé dans la tradition française (comme en témoignent, par exemple, les œuvres de Jean Cavaillès et Albert Lautman), ce dialogue s'est aujourd'hui arrêté, au sein de la philosophie des mathématiques, à des enjeux comme par exemple le type d'objectivité propre aux structures mathématiques, ou limités à des enjeux locaux, tels que les seuls aspects fondationnels de la théorie des catégories dans son opposition supposée à la théorie des ensembles.

Une réflexion philosophique qui s'occuperait de champs plus variés de la pratique mathématique, bénéficierait de la réflexivité propre des mathématiciens et étendrait les analyses existantes, reste encore à faire. Ces dernières années au sein de l'Institut de Mathématiques de Toulouse, en partenariat avec le laboratoire SPHERE de l'université Paris Diderot, historiens, philosophes et mathématiciens ont étudié ensemble de nouveaux champs de la pratique mathématique contemporaine auxquels les derniers contribuent, comme la géométrie algébrique ou la combinatoire, en particulier lors des deux écoles thématiques CNRS «Mathématiques et philosophie contemporaines» qui se sont tenues à Saint-Flour du 25 au 29 juin 2012 et du 3 au 7 juin 2013.

Ce numéro des Annales de la Faculté des Sciences de Toulouse offre un nouveau jalon pour ces travaux communs en présentant quatre perspectives complémentaires sur la théorie de l'homotopie, respectivement historique, philosophique, logique et mathématique. Il rassemble des articles tirés d'exposés donnés lors du Workshop Homotopie qui s'est tenu à l'Institut de Mathématiques de Toulouse du 20 au 21 octobre 2011.

La théorie de l'homotopie est l'un des développements majeurs de la topologie algébrique, et cristallise de façon exemplaire l'interaction des deux versants, algébrique et topologique, de cette discipline. Elle pousse à son terme le codage algébrique de données topologiques et donne lieu, en retour, à l'extension de méthodes topologiques à des contextes algébriques nouveaux (par exemple, celui des algèbres différentielles graduées). La théorie des catégories jouent un rôle fondamental dans ce double processus : la recherche de ce qui vaut «à équivalence d'homotopie près », qui caractérise bon nombre de constructions et de résultats en topologie algébrique, rejoint la recherche d'une présentation intrinsèque, telle que l'autorise la théorie des catégories 
(ainsi, la notion de catégorie de modèles permet de formaliser l'homotopie sans présupposer de segment unité). L'alliage de ces deux visées est sans doute l'un des traits caractéristiques de la théorie de l'homotopie, et c'est notamment ce que montrent, chacun à sa façon, les quatre articles de ce numéro.

La théorie de l'homotopie a récemment reçu une attention renouvelée dans le cadre du puissant programme de recherche initié par Vladimir Voevodsky, Steve Awodey et Thierry Coquand et intitulé «Univalent Foundations », dont le point de départ sont les liens dégagés entre la théorie des types de Per Martin-Löf et la théorie des catégories de modèles (ces liens permettent, pour simplifier, de penser deux preuves d'un même théorème sur le modèle de deux chemins homotopes). Dans ce numéro, d'autres éclairages de la théorie de l'homotopie sont proposés, complémentaires de ce programme, qui tâchent notamment d'en restituer toute la profondeur historique et l'ancrage dans la conceptualité mathématique.

Dans son article «The set of paths in a space and its algebraic structure: a historical account», Ralf Krömer se focalise sur un phénomène «retard» dans l'histoire du structuralisme : alors que l'étude du groupe fondamental des classes d'homotopie des lacets d'un espace connut un grand succès et fit l'objet de nombreux développements dans la première moitié du vingtième siècle, la notion plus générale de groupoïde fondamental ainsi que des relations d'équivalence plus fines que l'homotopie ne furent introduites puis étudiées que bien plus tardivement.

Dans une première partie, Krömer rappelle les quatre différentes approches du groupe fondamental successivement proposées par Henri Poincaré entre 1883 et 1908, avant de se tourner vers les travaux d'inspiration structuraliste de Hermann Weyl, Otto Schreier et Kurt Reidemeister mettant l'accent à la fois sur l'interaction fructueuse entre groupe fondamental et recouvrement universel via le groupe d'automorphismes associé, ainsi que sur des aspects proprement structuraux, tels que les sous-groupes et les groupes quotients du groupe fondamental, étudiés par exemple par Reidemeister dans le cadre de sa théorie des groupes de nœuds.

Krömer décrit ensuite la genèse des notions de groupoïde chez Heinrich Brandt et de pseudo-groupe de transformations chez Oswald Veblen et Henry Whitehead, l'introduction du groupoïde fondamental chez Normann Steenrod en relation avec les systèmes locaux et l'axiomatisation des théories homotopiques, avant d'étudier les traces qu'on retrouve de cette notion dans les travaux non publiés de Bourbaki.

Dans une dernière partie, Krömer analyse la transformation graduelle de l'intuition de la déformation continue des chemins en un concept rigoureux, 
grâce au changement de conception des chemins vus d'abord comme des ensembles de points puis comme des fonctions, et insiste sur le fait qu'une telle transition «permet [surtout] de traiter plus simplement et de généraliser le concept de déformation en le plongeant dans un riche cadre théorique (donné par la composition des flèches, dans les termes de la théorie des catégories)» (cf. p. 953). Suivant ce fil, l'auteur étudie ensuite les chemins homotopes définis sur un même ensemble de points obtenus par exemple au moyen d'un changement de paramètres, discute pour ce faire l'usage du terme «Weg» (chemin) chez Weyl et présente l'approche quelque peu différente de Max Newman. Comme on le sait bien, le passage des chemins à la classe d'équivalence des chemins homotopes est essentielle pour démontrer la propriété de groupe du groupe fondamental : c'est précisément ce qu'examine l'auteur en s'appuyant, entre autres, sur les manuels de Lew Pontrjagin et d'Edwin Spanier (écrit dans le langage des catégories).

L'article de Jean-Pierre Marquis, «Mathematical Models of Abstract Systems », a pour objet de comparer la construction des types d'homotopie, en topologie algébrique, avec la construction de modèles, au sens du travail de modélisation qu'on trouve dans les sciences appliquées. Cette comparaison n'est bien sûr pas une identification : la topologie algébrique fait un usage spécifique de modèles, qui s'explique par les objets et les visées propres à cette théorie. L'article veille à la fois à introduire la notion générale de modèle à propos des mathématiques, et à restituer la spécificité de la théorie de l'homotopie. Suivant une suggestion de David Mumford, Marquis défend en effet la thèse que certains objets mathématiques servent à en modéliser d'autres, et qu'on en rencontre particulièrement en topologie algébrique, sous les espèces des types d'homotopie.

L'article commence par expliquer le besoin de modéliser les espaces topologiques et présente pour cela une mise en perspective historique de la topologie algébrique, en expliquant les difficultés rencontrées dans la recherche des classes d'homéomorphismes entre espaces : ces difficultés ont montré qu'un cadre ensembliste n'était pas adéquat, et ont précipité la considération de complexes, c'est-à-dire l'émergence de la topologie combinatoire. Les modèles qu'on trouve en topologie algébrique sont bien des modèles au sens d'artefacts construits à chaque fois dans un but précis (dans le but de représenter un aspect ou une propriété précise). Marquis appelle modèle-objet (object model) d'un objet $\mathrm{Y}$ un objet $\mathrm{X}$ qui est construit selon une règle explicite à partir de $\mathrm{Y}$, qui possède toutes les propriétés pertinentes de $\mathrm{Y}$ dans le contexte en jeu, et dont la manipulation aisée, notamment en termes calculatoires, permet d'analyser efficacement Y. En particulier, X peut être un représentant commode du type d'homotopie de Y. De façon plus abstraite, la catégorie homotopique d'une catégorie donnée peut être 
comprise comme un modèle (au sens de ce que Marquis appelle un modèle systémique) de cette dernière.

La topologie algébrique s'est notamment développée en reconstruisant la plupart des espaces topologiques au moyen de combinaisons de cellules, appelées «complexes» : ces complexes sont comme tels des modèles d'espaces, dont les propriétés combinatoires facilitent la détermination d'invariants algébriques pour chaque espace modélisé. Comme le soutient Marquis, ces modèles ne sont pas les types d'homotopie eux-mêmes, mais des modes d'accès à ces types. Une des familles de modèles les plus centrales, en topologie algébrique, est celle des «CW-complexes». L'article détaille le concept de CW-complexe, ainsi que leur rôle dans la théorie de l'homotopie, tel que le révèle le théorème de Whitehead : les CW-complexes permettent de ramener l'équivalence d'homotopie aux invariants algébriques que constituent les groupes d'homotopie. Il reste que ces derniers sont très ardus à déterminer, d'où deux voies détaillées dans l'article.

La première voie consiste à introduire des types d'homotopie tronqués, ou $n$-types d'homotopie, puis des modèles des différents $n$-types : ainsi les ensembles modélisent les 0-types, et les groupoïdes modélisent les 1-types. Un modèle n'est plus alors homogène à l'espace qu'il modélise, il n'en donne plus une image, mais permet de codifier l'information nécessaire à la reconstruction d'un représentant du n-type d'homotopie concerné - représentant qui est lui-même, de son côté, un modèle d'espace. Cette première voie conduit naturellement à la théorie des catégories supérieures. Le point essentiel retenu par Marquis est la distinction entre deux genres de modélisations : la modélisation d'un espace par un CW-complexe (qui est lui-même un espace), la modélisation d'un $n$-type d'homotopie par une entité algébrique (qui ne relève plus de la topologie classique, mais de la théorie des catégories). La seconde voie exposée dans l'article correspond à celle des catégories homotopiques et des catégories de modèles. Une catégorie de modèles est un certain univers de types d'homotopie, au sens où la structure interne de cette catégorie permet de définir un équivalent de la plupart des notions homotopiques. La théorie des catégories de modèles a ainsi permis de généraliser la théorie de l'homotopie au-delà d'un contexte proprement topologique. Dans ce contexte, les ensembles simpliciaux (également mentionnés dans l'article de B. Halimi) forment une catégorie de modèles de référence : une catégorie de modèles dont la catégorie homotopique est une modélisation exacte des types d'homotopie (d'espaces topologiques), autrement dit un «modèle systémique» de ces types, pour reprendre la terminologie de l'auteur. L'article est suivi d'un appendice qui expose quelques concepts essentiels de la topologie algébrique, ainsi que leurs reconstructions en théorie des catégories. 
Dans son article «Sur une application possible du concept d'homotopie à la théorie des modèles » Brice Halimi examine le transfert de concepts et de structures de la théorie de l'homotopie à la théorie des modèles. Il prolonge ainsi une tradition bien établie d'exportation, en direction de la logique mathématique, de méthodes venues des mathématiques classiques (ainsi la dualité de Stone constitue-t-elle un instrument de circulation entre logique et topologie générale) et donne un nouvel exemple des rapports entre théorie des modèles et théorie de l'homotopie après les développements récents du programme «Univalent foundations of Mathematics» proposé par Vladimir Voevodsky.

L'enjeu pour l'auteur est «d'établir les premiers éléments d'une forme de dictionnaire de la théorie des modèles vers la théorie de l'homotopie, et de proposer un panorama d'applications possibles de la seconde à la première » (cf. p. 1019). Le point de départ de ce dictionnaire est syntaxique : il consiste en l'analogie permettant de comparer les formules d'un langage du premier ordre à des chaînes (au sens d'un complexe de chaînes en homologie) et un quantificateur unaire à un opérateur de face, et par suite de transposer la notion de bord à la logique du premier ordre. Cette perspective permet également de recomprendre la notion de séquent.

Halimi prolonge sémantiquement ce point de départ en montrant qu'à toute structure d'interprétation d'un langage du premier ordre peut être associé un ensemble simplicial via une correspondance fonctorielle traduisant les plongements élémentaires en morphismes d'ensembles simpliciaux (cf. théorème 3.1 p. 1031). Toute structure du premier ordre peut ainsi apparaître comme le représentant d'un type d'homotopie. Cette description en termes simpliciaux des modèles de n'importe quelle théorie du premier ordre est étendue aux espaces de types d'un modèle d'une théorie complète $T$. Non seulement l'ensemble des $n$-types, pour chaque $n \in \mathbb{N}$, peut naturellement être muni d'une topologie, mais la famille de ces ensembles donne ellemême lieu à un ensemble simplicial dont la réalisation est un nouvel espace topologique associé à $T$. Enfin, l'auteur montre que la «définition » d'un $n$ type peut être réinterprétée sous la forme d'un opérateur de différenciation.

Dans la mesure où les modèles d'une théorie du premier ordre $T$ correspondent à des ensembles simpliciaux et que la catégorie des ensembles simpliciaux est un exemple paradigmatique de «catégorie de modèles » (au sens homotopique), il est naturel d'envisager la catégorie des modèles de $T$ comme une pré-catégorie de modèles. C'est la piste explorée par l'article pour finir.

À l'aune du propos général de ce numéro spécial, l'auteur apporte ainsi un nouveau témoignage du caractère éminemment transversal de la théorie de l'homotopie en mathématiques. 
L'article de Tim Porter, «Variations on a theme of homotopy », s'attache, pour commencer, à l'idée d'identification : identifier, en topologie algébrique, c'est reconnaître, au sein d'un éventail d'espaces «vraiment différents », et au moyen d'invariants algébrico-géométriques, à quel type d'espace appartient tel ou tel espace étudié. Mais identifier, c'est aussi rendre deux objets identiques (par passage au quotient). Le point de départ développé par l'article est qu' «une homotopie est une raison pour identifier», dans les deux sens qui viennent d'être distingués. Porter insiste sur les outils fournis par la théorie abstraite de l'homotopie, et en particulier sur la notion de foncteur cylindre pour une catégorie. Cette dernière notion permet en effet de définir une notion élargie d'homotopie entre morphismes d'une catégorie, dès lors, justement, que cette catégorie est munie d'un foncteur cylindre. Cette notion élargie ne garantit ni la symétrie, ni la transitivité de la relation d'homotopie. La symétrie de la relation d'homotopie correspond à l'existence d'une involution sur le foncteur cylindre, la transitivité correspond quant à elle à l'existence d'une subdivision sur ce même foncteur cylindre : dans les deux cas, il s'agit donc d'une structure supplémentaire. propriétés de la relation d'homotopie dans une catégorie correspondent ainsi à l'existence de structures supplémentaires dans cette catégorie, et de superstructures sur ces structures.

Ce codage de propriétés par des foncteurs présente l'avantage de donner naturellement lieu à des itérations. La relation d'homotopie peut ainsi être redoublée en une notion de 2-homotopie, correspondant à des «raisons d'ordre supérieur », c'est-à-dire des raisons pour identifier des raisons pour identifier deux espaces. Porter analyse la notion résultante d'homotopie supérieure dans le cadre des ensembles cubiques, puis dans celui des ensembles simpliciaux (en décrivant l'un comme l'autre de ces deux cadres). Les conditions de cohérence homotopique (commutativité d'un diagramme de niveau $n$ à homotopie de niveau $n+1$ près) font ensuite l'objet d'une introduction à part entière.

La dernière partie de l'article est consacrée à une étude de cas, dans le but de corriger l'image intuitive de l'homotopie (comme déformation), et de montrer concrètement le besoin, dans certaines situations, d'outils (venus de l'homologie et de la shape theory) qui outrepassent cette image. En particulier, la pro-homotopy theory (ou théorie homotopique de catégories de pro-objets), dans le prolongement de l'homotopie étale, consiste en l'extension de la théorie classique de l'homotopie à des systèmes inverses d'espaces conçus comme des approximations de plus en plus fines d'un certain type d'espace. Elle pose notamment le problème du rapport entre l'existence d'une famille d'homotopies entre systèmes inverses, et l'existence d'une équivalence d'homotopie entre les deux types d'espaces approximés. 
La théorie de l'homotopie dirigée se rattache au même genre d'extension de la théorie classique. C'est ainsi après un panorama raisonné des différents développements contemporains en théorie de l'homotopie que se termine l'article.

Brice Halimi ${ }^{1}$, Sébastien Maronne ${ }^{2}$, Damian RössleR ${ }^{3}$

Toulouse, Décembre 2013

(1) Université Paris Ouest Nanterre La Défense, Département de Philosophie, 200, avenue de la République, 92001 Nanterre cedex. bhalimi@u-paris10.fr

(2) Institut de Mathématiques de Toulouse, Université Paul Sabatier, 118 route de Narbonne, F-31062 Toulouse Cedex 9. smaronne@math.univ-toulouse.fr

(3) Institut de Mathématiques de Toulouse, Université Paul Sabatier, 118 route de Narbonne, F-31062 Toulouse Cedex 9.

rossler@math.univ-toulouse.fr 